\title{
Intensity and Coherence Characteristics of a Radial Phase-Locked Multi-Gaussian Schell-Model Vortex Beam Array in Atmospheric Turbulence
}

\author{
Jialu Zhao, Guiqiu Wang, Xiaolu Ma, Haiyang Zhong, Hongming Yin, Yaochuan Wang and Dajun Liu * \\ Department of Physics, College of Science, Dalian Maritime University, Dalian 116026, China; \\ zj1970929@dlmu.edu.cn (J.Z.); gqwang@dlmu.edu.cn (G.W.); maxiaolu@dlmu.edu.cn (X.M.); \\ haae@dlmu.edu.cn (H.Z.); hmyin@dlmu.edu.cn (H.Y.); ycwang@dlmu.edu.cn (Y.W.) \\ * Correspondence: liudajun@dlmu.edu.cn; Tel.: +86-159-4111-5638
}

check for updates

Citation: Zhao, J.; Wang, G.; Ma, X.; Zhong, H.; Yin, H.; Wang, Y.; Liu, D Intensity and Coherence

Characteristics of a Radial

Phase-Locked Multi-Gaussian Schell-Model Vortex Beam Array in Atmospheric Turbulence. Photonics 2021, 8, 5. https://doi.org/10.3390/ photonics 8010005

Received: 19 November 2020 Accepted: 24 December 2020 Published: 29 December 2020

Publisher's Note: MDPI stays neutral with regard to jurisdictional clai$\mathrm{ms}$ in published maps and institutional affiliations.

Copyright: (C) 2020 by the authors. Licensee MDPI, Basel, Switzerland. This article is an open access article distributed under the terms and conditions of the Creative Commons Attribution (CC BY) license (https:// creativecommons.org/licenses/by/ $4.0 /)$.
Abstract: The theoretical descriptions for a radial phase-locked multi-Gaussian Schell-model vortex (RPLMGSMV) beam array is first given. The normalized intensity and coherence distributions of a RPLMGSMV beam array propagating in free space and atmospheric turbulence are illustrated and analyzed. The results show that a RPLMGSMV beam array with larger total number $N$ or smaller coherence length $\sigma$ can evolve into a beam with better flatness when the beam array translating into the flat-topped profile at longer distance $z$ and the flatness of the flat-topped intensity distribution can be destroyed by the atmospheric turbulence at longer distance $z$. The coherence distribution of a RPLMGSMV beam array in atmospheric turbulence at the longer distance will have Gaussian distribution. The research results will be useful in free space optical communication using a RPLMGSMV beam array.

Keywords: average intensity; multi-Gaussian Schell-model source; vortex beam; beam array; atmospheric turbulence

\section{Introduction}

With the development of wireless optical communication and laser radar, the evolutions of laser beams in atmospheric turbulence were widely studied in past years [1]. In past years, the properties of fully coherent laser beams in turbulence have been widely analyzed, such as Gaussian beams [2], Hermite-Gaussian beam [3], Pearcey-Gaussian beam [4], beam array [5-7], Laguerre-Gaussian beam [8], Airy beam [9], hollow beam [10,11], vortex beam [12,13], and vortex lattices [14]. From previous studies [1], one can see that partially coherent beams are resistance to the deleterious effects of turbulence. In past years, the properties, including intensity, polarization, and coherence, of Gaussian Schell-model (GSM) beams [15-19] and GSM beam array [20-23] propagating in turbulence were widely studied. On the other hand, the special correlated beams are also be introduced and analyzed, such as non-uniformly correlated beams [24], multi-Gaussian Schell-model (MGSM) beams [25-28], cosine-GSM beams [29], multi-cosine-Laguerre-Gaussian correlated Schellmodel beams [30]. Laser arrays can produce the higher power output than single beam and which can have linear, rectangular and radial distributions. The beams correlated with MGSM source can provide flat intensity profiles in the far field [25]. To obtain the flat-topped intensity profiles, the MGSM beam arrays propagating in turbulence are investigated, and it is found that the MGSM beam arrays can achieve the better flat-topped profiles [31,32]. Moreover, the MGSM vortex beam has been introduced and studied. It shows that the intensity profile of MGSM vortex can be modulated by the topological charge [33]. Thus, it will be very interesting to consider the laser array composed by MGSM vortex beams. In this paper, we extend MGSM vortex beam into the radial phaselocked multi-Gaussian-Schell-model vortex (RPLMGSMV) beam array, and investigate 
the intensity and coherence properties of RPLMGSMV beam array propagating in free space and atmospheric turbulence. Moreover, the model of laser arrays with linear and rectangular distributions can also be obtained in the similar analytical approach.

\section{Theory Analysis}

\subsection{Analytical Description of RPLMGSMV Beam Array}

The electric field distribution of a Gaussian vortex beam at source plane $z=0$ is described by

$$
E\left(\mathbf{r}_{0}, 0\right)=\left[x+i \operatorname{sgn}(M) y_{0}\right]^{|M|} \exp \left(-\frac{x_{0}^{2}+y_{0}^{2}}{w_{0}^{2}}\right)
$$

where $w_{0}$ is beam waist and $M$ is the topological charge.

In this work, laser array with radial distribution will be analyzed as example, the electric field of a radial phase-locked Gaussian vortex beam array with $Q$ beamlets can be given as:

$$
E_{Q}\left(\mathbf{r}_{0}, 0\right)=\sum_{q=1}^{Q}\left[\left(x_{0}-r_{q x}\right)+i \operatorname{sgn}(M)\left(y_{0}-r_{q y}\right)\right]^{|M|} \exp \left[-\frac{\left(x_{0}-r_{q x}\right)^{2}+\left(y_{0}-r_{q y}\right)^{2}}{w_{0}^{2}}\right] \exp \left(i \varphi_{q}\right)
$$

with

$$
r_{q x}=R \cos \varphi_{q}, r_{q y}=R \sin \varphi_{q}, \varphi_{q}=q \frac{2 \pi}{Q}, q=1,2, \cdots Q
$$

where $R$ is radius; $\varphi_{n}$ is the phase of the $q$-th beamlet; $r_{q x}$ and $r_{q y}$ are the center of the $q$-th beamlet element located at $z=0$.

Considering the unified theory of coherence and polarization [34], the cross spectral density (CSD) of partially coherent beams can be expressed as

$$
W\left(\mathbf{r}_{10}, \mathbf{r}_{20}\right)=\left\langle E\left(\mathbf{r}_{10}\right) E^{\times}\left(\mathbf{r}_{20}\right)\right\rangle
$$

Introducing a MGSM correlation [22], the CSD of a RPLMGSMV beam array with $Q$ beamlets can be written as

$$
\begin{aligned}
W \quad & \left(\mathbf{r}_{10}, \mathbf{r}_{20}, 0\right)=\sum_{q_{1}=1}^{Q} \sum_{q_{2}=1}^{Q} \exp \left[i\left(\varphi_{q 1}-\varphi_{q 2}\right)\right] \\
& \times\left[\left(x_{10}-r_{q 1 x}\right)+i \operatorname{sgn}(M)\left(y_{10}-r_{q 1 y}\right)\right]^{|M|} \exp \left[-\frac{\left(x_{10}-r_{q 1 x}\right)^{2}+\left(y_{10}-r_{q 1 y}\right)^{2}}{w_{0}^{2}}\right] \\
& \times\left[\left(x_{20}-r_{q 2 x}\right)-i \operatorname{sgn}(M)\left(y_{20}-r_{q 2 y}\right)\right]^{|M|} \exp \left[-\frac{\left(x_{20}-r_{q 2 x}\right)^{2}+\left(y_{20}-r_{q 2 y}\right)^{2}}{w_{0}^{2}}\right] \\
& \times \frac{1}{C_{0}} \sum_{n=1}^{N}\left(\begin{array}{c}
N \\
n
\end{array}\right) \frac{(-1)^{n-1}}{n} \exp \left\{-\frac{\left[\left(x_{10}-r_{q 1 x}\right)-\left(x_{20}-r_{q 2 x}\right)\right]^{2}}{2 n \sigma^{2}}-\frac{\left[\left(y_{10}-r_{q 1 y}\right)-\left(y_{20}-r_{q 2 y}\right)\right]^{2}}{2 n \sigma^{2}}\right\}
\end{aligned}
$$

where $N$ is total number of terms of MGSM source, $\sigma$ is the coherence length, $C_{0}$ is the normalized factor, and can be described by

$$
C_{0}=\sum_{n=1}^{N}\left(\begin{array}{c}
N \\
n
\end{array}\right) \frac{(-1)^{n-1}}{n}
$$

Figure 1 shows the normalized intensity of a RPLMGSMV beam array at $z=0$ for the different $Q$, one can see that the beamlets of a RPLMGSMV beam array have the hollow center. 
(a)

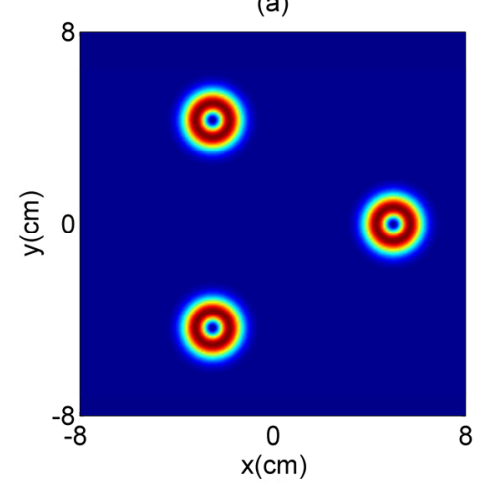

(c)

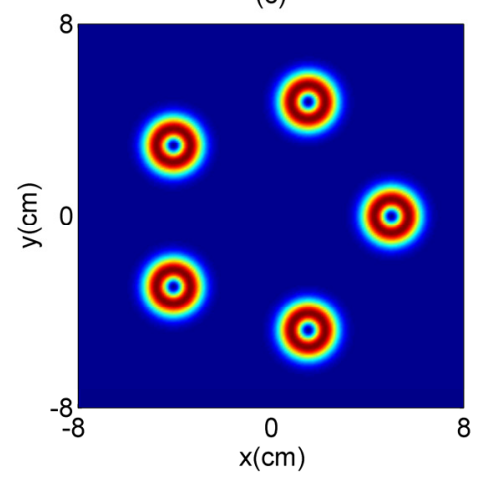

(b)

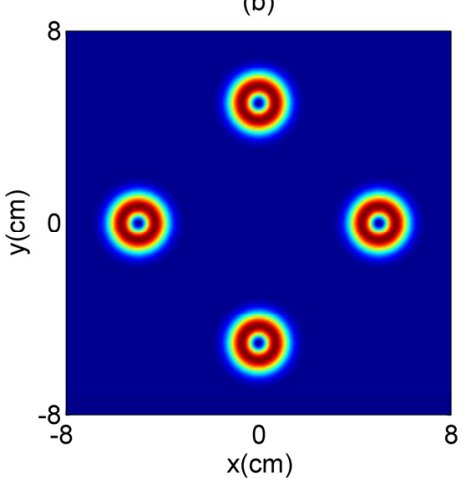

(d)

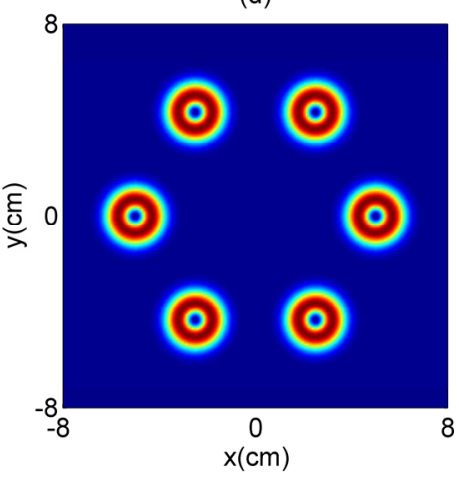

Figure 1. Normalized intensity of a RPLMGSMV beam array at $z=0$ for the different $Q$. (a) $Q=3$, (b) $Q=4$, (c) $Q=5$, (d) $Q=6$.

\subsection{Propagation Analysis}

Based on the extended Huygens-Fresnel integral, the CSD of a RPLMGSMV beam array propagating in atmospheric turbulence at plane $z$ can be read as [1]

$$
\begin{gathered}
W \quad\left(\mathbf{r}_{1}, \mathbf{r}_{2}, z\right)=\frac{k^{2}}{4 \pi^{2} z^{2}} \int_{-\infty}^{+\infty} \int_{-\infty}^{+\infty} \int_{-\infty}^{+\infty} \int_{-\infty}^{+\infty} W\left(\mathbf{r}_{10}, \mathbf{r}_{20}, 0\right) \\
\times \exp \left[-\frac{i k}{2 z}\left(\mathbf{r}_{1}-\mathbf{r}_{10}\right)^{2}+\frac{i k}{2 z}\left(\mathbf{r}_{2}-\mathbf{r}_{20}\right)^{2}\right] \\
\left.\quad \times \exp \left[\psi\left(\mathbf{r}_{10}, \mathbf{r}_{1}\right)+\psi^{*}\left(\mathbf{r}_{20}, \mathbf{r}_{2}\right)\right]\right\rangle d \mathbf{r}_{10} d \mathbf{r}_{20}
\end{gathered}
$$

with

$$
\left\langle\exp \left[\psi\left(\mathbf{r}_{10}, \mathbf{r}\right)+\psi^{*}\left(\mathbf{r}_{20}, \mathbf{r}\right)\right]\right\rangle=\exp \left[-\frac{\left(\mathbf{r}_{10}-\mathbf{r}_{20}\right)^{2}+\left(\mathbf{r}_{10}-\mathbf{r}_{20}\right)\left(\mathbf{r}_{1}-r_{2}\right)+\left(\mathbf{r}_{1}-\mathbf{r}_{2}\right)^{2}}{\rho_{0}^{2}}\right]
$$

In the above equation, the spatial coherence length $\rho_{0}$ can be expressed as

$$
\rho_{0}=\left(0.545 C_{n}^{2} k^{2} z\right)^{-3 / 5}
$$

where $C_{n}^{2}$ is the structure constant of atmospheric turbulence.

Substituting Equation (5) into Equation (7), the CSD of a RPLMGSMV beam array propagating in atmospheric turbulence at plane $z$ can be derived as

$$
\begin{aligned}
W \quad\left(\mathbf{r}_{1}, \mathbf{r}_{2}, z\right)= & \sum_{q_{1}=1}^{Q} \sum_{q_{2}=1}^{Q} \exp \left[i\left(\varphi_{q 1}-\varphi_{q 2}\right)\right] \frac{k^{2}}{4 \pi^{2} z^{2}} \exp \left[-\frac{i k}{2 z}\left(\mathbf{r}_{1}^{2}-\mathbf{r}_{2}^{2}\right)\right] \exp \left[-\frac{\left(x_{1}-x_{2}\right)^{2}+\left(y_{1}-y_{2}\right)^{2}}{\rho_{0}^{2}}\right] \\
& \times \frac{1}{C_{0}} \sum_{l_{1}=0}^{|M|} \frac{|M| ! l_{1}}{l_{1} !\left(|M|-l_{1}\right) !} \sum_{l_{2}=0}^{|M|} \frac{|M| !(-i)^{l_{2}}}{l_{2} !\left(|M|-l_{2}\right) !} \sum_{n=1}^{N}\left(\begin{array}{c}
N \\
n
\end{array}\right) \frac{(-1)^{n-1}}{n} W(x, z) W(y, z)
\end{aligned}
$$


with

$$
\begin{aligned}
& W \quad(x, z)=\exp \left[-\frac{1}{\rho_{0}^{2}}\left(r_{q_{1} x}-r_{q_{2} x}\right)^{2}\right] \exp \left[-\frac{i k}{2 z}\left(r_{q_{1} x}^{2}-r_{q_{2} x}^{2}\right)\right] \exp \left[2 \frac{i k}{2 z}\left(x_{1} r_{q_{1} x}-x_{2} r_{q_{2} x}\right)\right] \\
& \times \exp \left[-\frac{\left(x_{1}-x_{2}\right)\left(r_{q_{1} x}-r_{q_{2} x}\right)}{\rho_{0}^{2}}\right] \sqrt{\frac{\pi}{a}}\left(|M|-l_{1}\right) !\left(\frac{1}{a}\right)^{|M|-l_{1}} \\
& \times \exp \left[\frac{1}{a}\left(\frac{i k}{2 z} x_{1}-\frac{i k}{2 z} r_{q_{1} x}-\frac{x_{1}-x_{2}+2\left(r_{q_{1} x}-r_{q_{2}} x\right)}{2 \rho_{0}^{2}}\right)^{2}\right]\left[\frac{\left[M \mid-l_{1}\right.}{\left.\sum_{k_{1}=0}^{2}\right]} \frac{1}{k_{1} !\left(|M|-l_{1}-2 k_{1}\right) !}\left(\frac{a}{4}\right)^{k_{1}}\right. \\
& \times \sum_{s=0}^{|M|-l_{1}-2 k_{1}} \frac{\left(|M|-l_{1}-2 k_{1}\right) !}{s_{1} !\left(|M|-l_{1}-2 k_{1}-s_{1}\right) !}\left(\frac{i k}{2 z} x_{1}-\frac{i k}{2 z} r_{q_{1} x}-\frac{x_{1}-x_{2}+2\left(r_{q_{1} x}-r_{q_{2} x}\right)}{2 \rho_{0}^{2}}\right)^{|M|-l_{1}-2 k_{1}-s_{1}}\left(\frac{1}{2 n \sigma^{2}}+\frac{1}{\rho_{0}^{2}}\right)^{s_{1}} \\
& \times \sqrt{\frac{\pi}{b}}\left(\frac{i}{2 \sqrt{b}}\right)^{|M|-l_{2}+s_{1}} \exp \left(\frac{c_{x}^{2}}{b}\right) H_{|M|-l_{2}+s_{1}}\left(-\frac{i c_{x}}{\sqrt{b}}\right) \\
& W \quad(y, z)=\exp \left[-\frac{1}{\rho_{0}^{2}}\left(r_{q_{1} y}-r_{q_{2} y}\right)^{2}\right] \exp \left[-\frac{i k}{2 z}\left(r_{q_{1} y}^{2}-r_{q_{2} y}^{2}\right)\right] \exp \left[2 \frac{i k}{2 z}\left(y_{1} r_{q_{1} y}-y_{2} r_{q_{2} y}\right)\right] \\
& \times \exp \left[-\frac{\left(y_{1}-y_{2}\right)\left(r_{q_{1}}-r_{q_{2} y}\right)}{\rho_{0}^{2}}\right] \sqrt{\frac{\pi}{a}}\left(l_{1}\right) !\left(\frac{1}{a_{y}}\right)^{l_{1}} \\
& \times \exp \left[\frac{1}{a_{y}}\left(\frac{i k}{2 z} y_{1}-\frac{i k}{2 z} r_{q_{1} y}-\frac{y_{1}-y_{2}+2\left(r_{q_{1}}-r_{q_{2} y}\right)}{2 \rho_{0}^{2}}\right)^{2}\right] \sum_{k_{2}=0}^{\left[\frac{l_{1}}{2}\right]} \frac{1}{k_{2} !\left(l_{1}-2 k_{2}\right) !}\left(\frac{a}{4}\right)^{k_{2}} \\
& \times \sum_{s_{2}=0}^{l_{1}-2 k_{2}} \frac{\left(l_{1}-2 k_{2}\right) !}{s_{3} !\left(l_{1}-2 k_{2}-s_{2}\right) !}\left(\frac{i k}{2 z} y_{1}-\frac{i k}{2 z} r_{q_{1} y}-\frac{y_{1}-y_{2}+2\left(r_{q_{1} y}-r_{q_{2} y}\right)}{2 \rho_{0}^{2}}\right)^{l_{1}-2 k_{2}-s_{2}}\left(\frac{1}{2 n \sigma^{2}}+\frac{1}{\rho_{0}^{2}}\right)^{s_{2}} \\
& \times \sqrt{\frac{\pi}{b}}\left(\frac{i}{2 \sqrt{b}}\right)^{l_{2}+s} \exp \left(\frac{c_{y}^{2}}{b}\right) H_{l_{2}+s_{2}}\left(-\frac{i c_{y}}{\sqrt{b}}\right)
\end{aligned}
$$

where

$$
\begin{gathered}
a=\frac{1}{w_{0}^{2}}+\frac{1}{2 n \sigma^{2}}+\frac{1}{\rho_{0}^{2}}+\frac{i k}{2 z} \\
b=\frac{1}{w_{0}^{2}}+\frac{1}{\rho_{0}^{2}}+\frac{1}{2 n \sigma^{2}}-\frac{i k}{2 z}-\frac{1}{a}\left(\frac{1}{2 n \sigma^{2}}+\frac{1}{\rho_{0}^{2}}\right)^{2} \\
c_{x}=\frac{i k}{2 z} r_{q_{2} x}-\frac{i k}{2 z} x_{2}+\frac{x_{1}-x_{2}+2\left(r_{q_{1} x}-r_{q_{2} x}\right)}{2 \rho_{0}^{2}} \\
+\frac{1}{a}\left(\frac{1}{2 n \sigma^{2}}+\frac{1}{\rho_{0}^{2}}\right)\left[\frac{i k}{2 z} x_{1}-\frac{i k}{2 z} r_{q_{1} x}-\frac{x_{1}-x_{2}+2\left(r_{q_{1} x}-r_{q_{2} x}\right)}{2 \rho_{0}^{2}}\right] \\
c_{y}=\frac{i k}{2 z} r_{q_{2} y}-\frac{i k}{2 z} y_{2}+\frac{y_{1}-y_{2}+2\left(r_{q_{1} y}-r_{q_{2} y}\right)}{2 \rho_{0}^{2}} \\
+\frac{1}{a}\left(\frac{1}{2 n \sigma^{2}}+\frac{1}{\rho_{0}^{2}}\right)\left[\frac{i k}{2 z} y_{1}-\frac{i k}{2 z} r_{q_{1} y}-\frac{y_{1}-y_{2}+2\left(r_{q_{1} y}-r_{q_{2} y}\right)}{2 \rho_{0}^{2}}\right]
\end{gathered}
$$

In the derivations of Equation (10), the following equations has been applied [35]

$$
\begin{aligned}
& (x+i y)^{M}=\sum_{l=0}^{M} \frac{M ! i^{l}}{l !(M-l) !} x^{M-l} y^{l} \\
& \int_{-\infty}^{+\infty} x^{n} \exp \left(-a x^{2}+2 b x\right) d x=\sqrt{\frac{\pi}{a}}\left(\frac{i}{2 \sqrt{a}}\right)^{n} \exp \left(\frac{b^{2}}{a}\right) H_{n}\left(-\frac{i b}{\sqrt{a}}\right) \\
& H_{n}(x)=\sum_{l=0}^{\left[\frac{n}{2}\right]} \frac{(-1)^{l} n !}{l !(n-2 l) !}(2 x)^{n-2 l}
\end{aligned}
$$


When $\mathbf{r}_{1}=\mathbf{r}_{2}=\mathbf{r}$ in Equation (10), the intensity of a RPLMGSMV beam array propagating in atmospheric turbulence is written as

$$
\begin{aligned}
I(\mathbf{r}, z)= & \frac{k^{2}}{4 \pi^{2} z^{2}} \frac{1}{C_{0}} \sum_{q_{1}=1}^{Q} \sum_{q_{2}=1}^{Q} \sum_{l_{1}=0}^{|M|} \frac{|M| ![i \operatorname{sgn}(M)]_{1}^{l_{1}}}{l_{1} !\left(|M|-l_{1}\right) !} \sum_{l_{2}=0}^{|M|} \frac{|M| ![-i \operatorname{sgn}(M)]_{2}}{l_{2} !\left(|M|-l_{2}\right) !} \\
& \times \sum_{n=1}^{N}\left(\begin{array}{c}
N \\
n
\end{array}\right) \frac{(-1)^{n-1}}{n} \exp \left[i\left(\varphi_{q 1}-\varphi_{q 2}\right)\right] I(x, z) I(y, z)
\end{aligned}
$$

where

$$
\begin{aligned}
& I(x, z)=\exp \left[-\frac{1}{\rho_{0}^{2}}\left(r_{q_{1} x}-r_{q_{2} x}\right)^{2}\right] \exp \left[-\frac{i k}{2 z}\left(r_{q_{1} x}^{2}-r_{q_{2} x}^{2}\right)\right] \exp \left[2 \frac{i k}{2 z}\left(r_{q_{1} x}-r_{q_{2} x}\right) x\right] \\
& \sqrt{\frac{\pi}{a}}\left(|M|-l_{1}\right) !\left(\frac{1}{a}\right)^{|M|-l_{1}} \exp \left[\frac{1}{a}\left(\frac{i k}{2 z} x-\frac{i k}{2 z} r_{q_{1} x}-\frac{r_{q_{1} x}-r_{q_{2} x}}{\rho_{0}^{2}}\right)^{2}\right] \\
& \sum_{k_{1}=0}^{\left[\frac{|M|-l_{1}}{2}\right]} \frac{1}{k_{1} !\left(|M|-l_{1}-2 k_{1}\right) !}\left(\frac{a}{4}\right)^{k_{1}} \sum_{s_{1}=0}^{|M|-l_{1}-2 k_{1}} \frac{\left(|M|-l_{1}-2 k_{1}\right) !}{s_{1} !\left(|M|-l_{1}-2 k_{1}-s_{1}\right) !} \\
& \left(\frac{i k}{2 z} x-\frac{i k}{2 z} r_{q_{1} x}-\frac{r_{q_{1} x}-r_{q_{2} x}}{\rho_{0}^{2}}\right)^{|M|-l_{1}-2 k_{1}-s_{1}}\left(\frac{1}{2 n \sigma^{2}}+\frac{1}{\rho_{0}^{2}}\right)^{s_{1}} \\
& \sqrt{\frac{\pi}{b}}\left(\frac{i}{2 \sqrt{b}}\right)^{|M|-l_{2}+s_{1}} \exp \left(\frac{c_{x x}^{2}}{b}\right) H_{|M|-l_{2}+s_{1}}\left(-\frac{i c_{x x}}{\sqrt{b}}\right) \\
& I(y, z)=\exp \left[-\frac{1}{\rho_{0}^{2}}\left(r_{q_{1} y}-r_{q_{2} y}\right)^{2}\right] \exp \left[-\frac{i k}{2 z}\left(r_{q_{1} y}^{2}-r_{q_{2} y}^{2}\right)\right] \exp \left[2 \frac{i k}{2 z}\left(r_{q_{1} y}-r_{q_{2} y}\right) y\right] \\
& \sqrt{\frac{\pi}{a}} l_{1} !\left(\frac{1}{a}\right)^{l_{1}} \exp \left[\frac{1}{a}\left(\frac{i k}{2 z} y-\frac{i k}{2 z} r_{q_{1} y}-\frac{r_{q_{1} y}-r_{q_{2} y}}{\rho_{0}^{2}}\right)^{2}\right] \\
& \sum_{k_{2}=0}^{\left[\frac{l_{1}}{2}\right]} \frac{1}{k_{2} !\left(l_{1}-2 k_{2}\right) !}\left(\frac{a}{4}\right)^{k_{2}} \sum_{s_{2}=0}^{l_{1}-2 k_{2}} \frac{\left(l_{1}-2 k_{2}\right) !}{s_{2} !\left(l_{1}-2 k_{2}-s_{2}\right) !} \\
& \left(\frac{i k}{2 z} y-\frac{i k}{2 z} r_{q_{1} y}-\frac{r_{q_{1} y}-r_{q_{2} y}}{\rho_{0}^{2}}\right)^{l_{1}-2 k_{2}-s_{2}}\left(\frac{1}{2 n \sigma^{2}}+\frac{1}{\rho_{0}^{2}}\right)^{s_{2}} \\
& \sqrt{\frac{\pi}{b}}\left(\frac{i}{2 \sqrt{b}}\right)^{l_{2}+s_{2}} \exp \left(\frac{c_{y y}^{2}}{b}\right) H_{l_{2}+s_{2}}\left(-\frac{i c_{y y}}{\sqrt{b}}\right)
\end{aligned}
$$

with

$$
\begin{aligned}
& c_{x x}=\frac{i k}{2 z} r_{q_{2} x}-\frac{i k}{2 z} x+\frac{r_{q_{1} x}-r_{q_{2} x}}{\rho_{0}^{2}}+\frac{1}{a}\left(\frac{1}{2 n \sigma^{2}}+\frac{1}{\rho_{0}^{2}}\right)\left(\frac{i k}{2 z} x-\frac{i k}{2 z} r_{q_{1} x}-\frac{r_{q_{1} x}-r_{q_{2} x}}{\rho_{0}^{2}}\right) \\
& c_{y y}=\frac{i k}{2 z} r_{q_{2} y}-\frac{i k}{2 z} y+\frac{r_{q_{1} y}-r_{q_{2} y}}{\rho_{0}^{2}}+\frac{1}{a}\left(\frac{1}{2 n \sigma^{2}}+\frac{1}{\rho_{0}^{2}}\right)\left(\frac{i k}{2 z} y-\frac{i k}{2 z} r_{q_{1} y}-\frac{r_{q_{1} y}-r_{q_{2} y}}{\rho_{0}^{2}}\right)
\end{aligned}
$$

The degree of coherence for a RPLMGSMV beam array propagating in atmospheric turbulence at plane $z$ is given as [34]

$$
\mu\left(\mathbf{r}_{1}, \mathbf{r}_{2}, z\right)=\frac{W\left(\mathbf{r}_{1}, \mathbf{r}_{2}, z\right)}{\left[W\left(\mathbf{r}_{1}, \mathbf{r}_{1}, z\right) W\left(\mathbf{r}_{2}, \mathbf{r}_{2}, z\right)\right]^{1 / 2}}
$$

\section{Numerical Results and Discussions}

In this section, the intensity and coherence distributions of a RPLMGSMV beam array in free space will firstly be investigated, and then the influences of atmospheric turbulence 
on intensity and coherence distributions of a RPLMGSMV beam array will be discussed. The relevant parameters in numerical simulations are selected as $\lambda=532 \mathrm{~nm}, w_{0}=1 \mathrm{~cm}$, $\sigma=1 \mathrm{~mm}, N=10, M=1$ and $R=5 \mathrm{~cm}$ without other explanations.

The normalized intensity of a RPLMGSMV beam array with $Q=5$ in free space at the different distances is illustrated in Figure 2. As can be seen from Figures $2 \mathrm{a}$ and $1 \mathrm{c}$, the dark hollow center of beamlets of a RPLMGSMV beam array will evolve into a Gaussian-like beam at $z=50 \mathrm{~m}$ (Figure 2a), while the beamlets have the dark hollow center at $z=0$ (Figure 1c); The reason that the dark hollow profile translating into the Gaussian beam can be explained as the effect of initial coherence length [33]. As the $z$ increases further, the Gaussian-like beamlets can evolve into a beam with flat-topped profile (Figure 2b), and the beamlets will also begin to overlap with each other (Figure 2b); thus, a RPLMGSMV beam array can translate into a beam with Gaussian-like intensity distribution; at last, the RPLMGSMV beam array can evolve from beam array into the beam with flat-topped intensity distribution at longer distance $z$ (Figure $2 \mathrm{~d}$ ). The phenomenon whereby a RPLMGSMV evolves into a beam with flat-topped profile is dominated by MGSM correlated function, and similar evolutions can also be found in the previous reports [25-28,33].

(a)

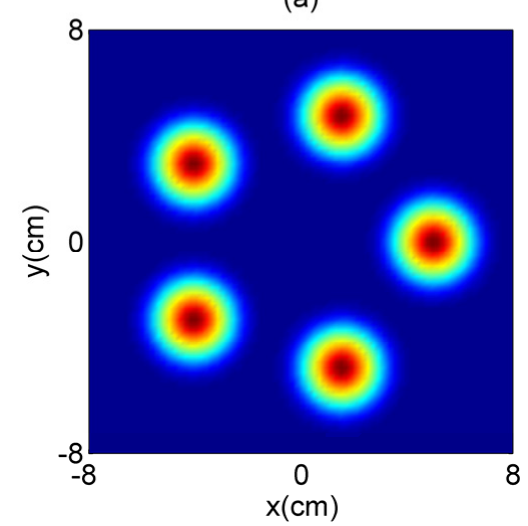

(c)

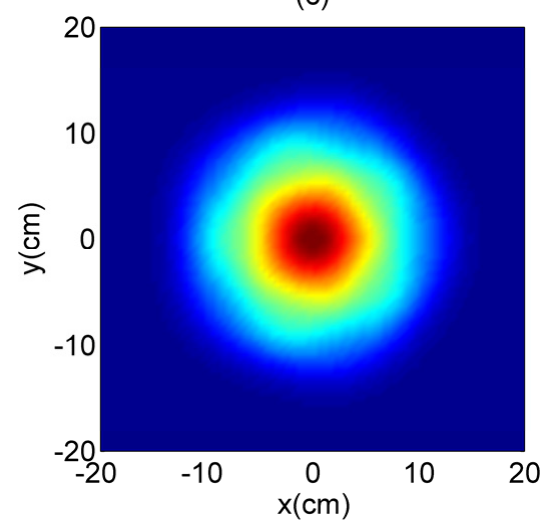

(b)

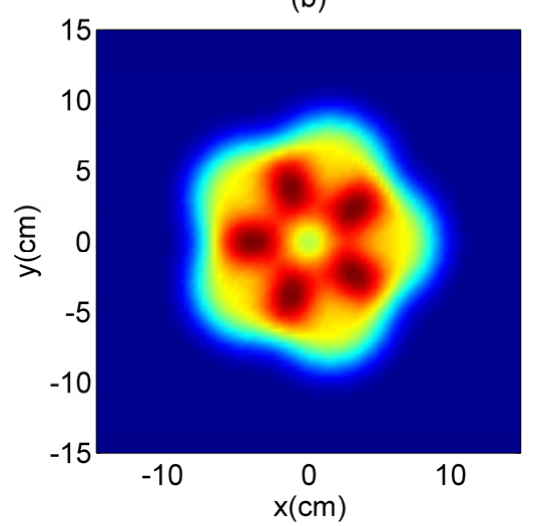

(d)

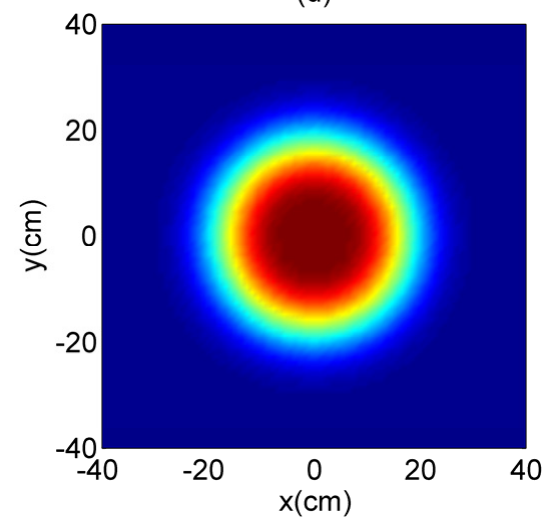

Figure 2. Normalized intensity of a RPLMGSMV beam array with $Q=5$ in free space. (a) $z=50 \mathrm{~m}$, (b) $z=200 \mathrm{~m},(\mathbf{c}) z=400 \mathrm{~m},(\mathbf{d}) z=900 \mathrm{~m}$.

To view the action of $Q$ on intensity distribution, normalized intensity of a RPLMGSMV beam array with $Q=4$ in free space are illustrated in Figure 3. As $z$ increases, it is found that the evolution of intensity distributions of a RPLMGSMV beam array with $Q=4$ are almost the same with a RPLMGSMV beam array with $Q=5$ (Figure 2), the beamlets of beam array will lose the dark hollow profile and become a beam with Gaussian-like beam distribution, the beam array with $Q=4$ will translate into the flat-topped profile (Figure 3b). Moreover, the flat-topped profile of a RPLMGSMV beam array is dominated by the MGSM sources at longer distance [33]. By comparing Figures 2 and 3, we can conclude 
that a RPLMGSMV beam array with the different $Q$ will evolve form beam array into flat-topped profile due to the action of MGSM source.

(a)

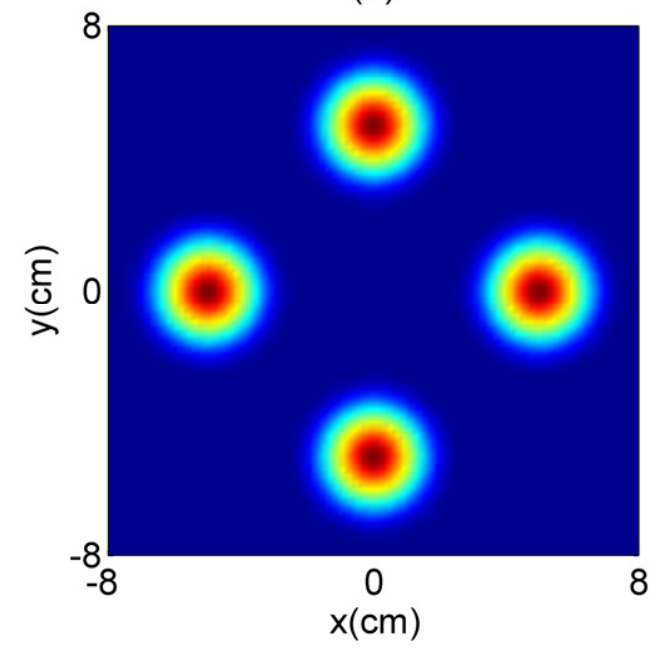

(b)

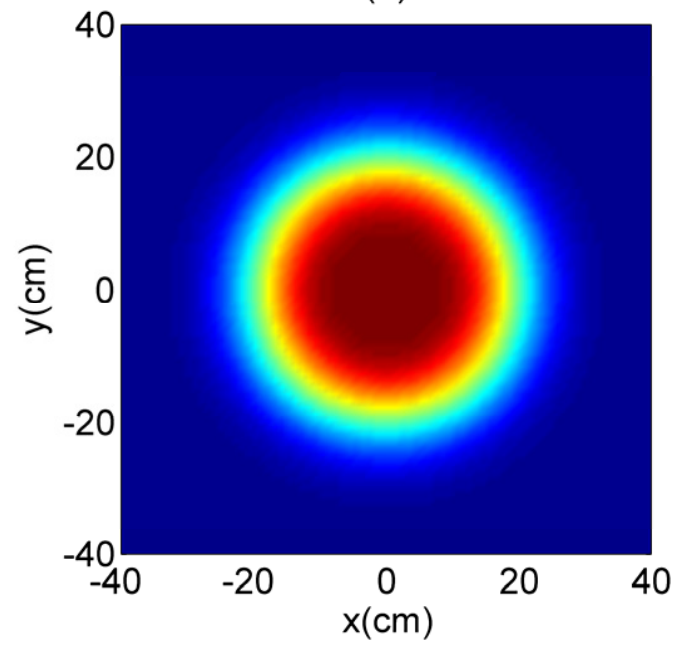

Figure 3. Normalized intensity of a RPLMGSMV beam array with $Q=4$ in free space. (a) $z=50 \mathrm{~m}$, (b) $z=1000 \mathrm{~m}$.

To view the effects of source parameters on the evolutions of intensity of a RPLMGSMV beam array propagating in free space, the cross sections $(y=0)$ of the normalized intensity of a RPLMGSMV beam array with $Q=4$ in free space for source parameters $\sigma, N$ and $M$ at the different distances are shown in Figures 4-6, respectively. From Figure 4, it is found that the RPLMGSMV beam array with larger $\sigma$ will lose the dark hollow distribution slower than the beam array with smaller $\sigma$. So, the smaller coherence length $\sigma$ will accelerate the evolutions of beam array translating into flat-topped profile, and the beam array with smaller $\sigma$ will have the better flatness when the beam array translating into flat-topped intensity profile at the longer distance $z$ (Figure $4 \mathrm{~d}$ ). Thus, it can conclude that the speed of a beam array translating into the flat-topped profile can be dominated by setting different $\sigma$ of MGSM source. Figure 5 shows that the RPLMGSMV beam array with larger $N$ will evolve from beam array into flat-topped beam faster, and which will have the better flatness when the beam array translating into the flat-topped beam at last (Figure 5d). The flatness of flat-topped profile is dominated by the total number $N$ of MGSM source, and the similar results can also be seen in the previous work [25]. Thus, from previous discussions, one can conclude that the flatness of flat-topped profile generating by RPLMGSMV beam array can be modulated by the parameters $\sigma$ and $N$ in the far field. The better the flatness of flat-topped profile is, the more power can be received by the same receiver, this is helpful for received power of free space optical communication. One can see from Figure 6 that the RPLMGSMV beam array with larger $M$ will have the larger dark hollow center at $z=0$ (Figure 6a), while the influences of different $M$ on the intensity distribution will disappear as the beam array evolve into the flat-topped beam at the longer distance $z$ (Figure $6 \mathrm{~d}$ ). Thus, we can conclude that the flat-topped profile is not correlated with topological charge in the far field. 
(a)

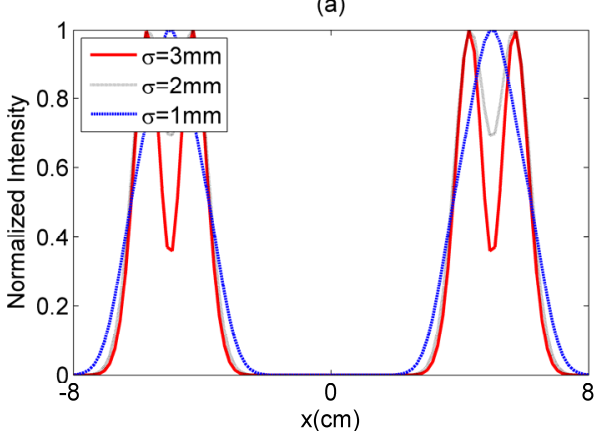

(c)

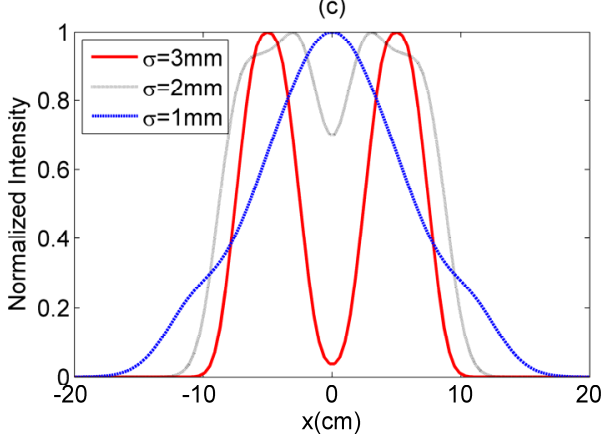

(b)
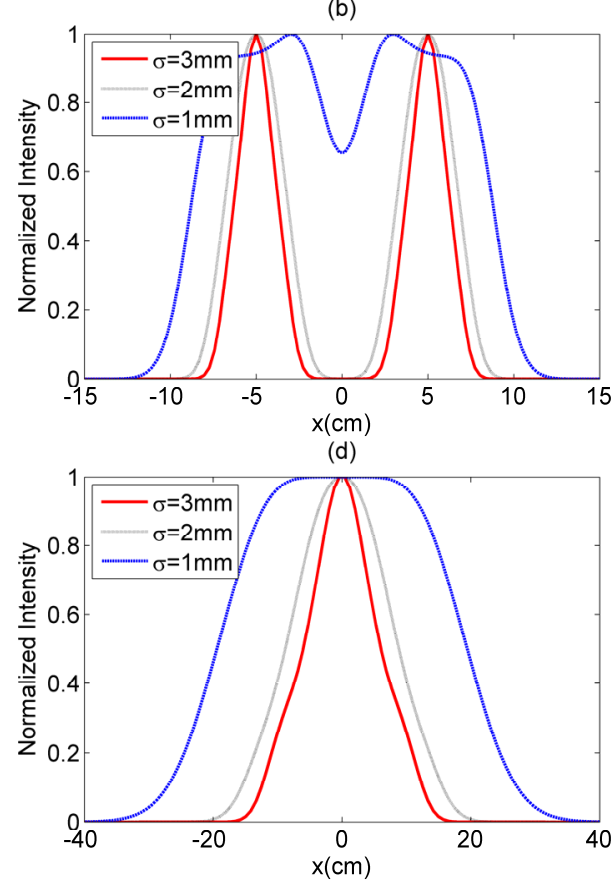

Figure 4. Cross sections $(y=0)$ of normalized intensity of a RPLMGSMV beam array with $Q=4$ in free space for the different $\sigma$. (a) $z=50 \mathrm{~m},(\mathbf{b}) z=200 \mathrm{~m},(\mathbf{c}) z=400 \mathrm{~m},(\mathbf{d}) z=1000 \mathrm{~m}$.

(a)

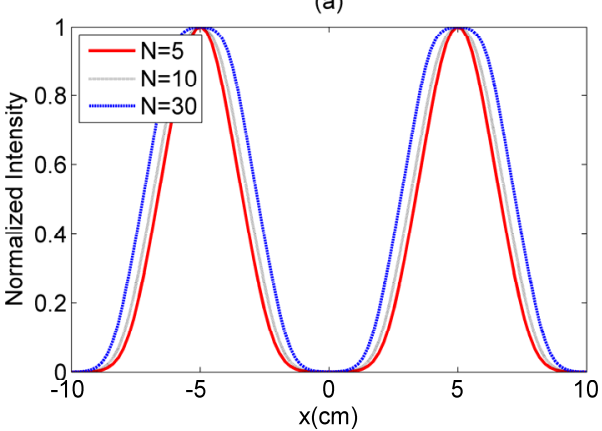

(c)

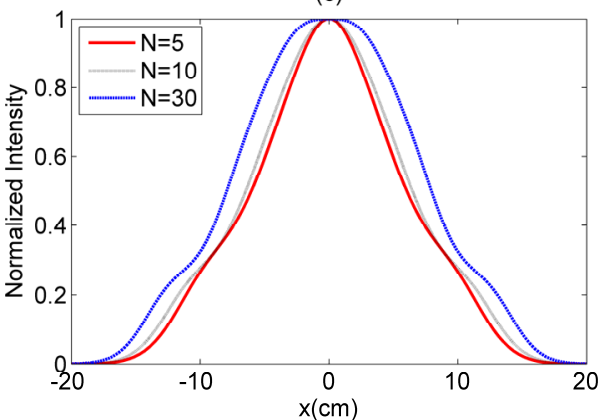

(b)
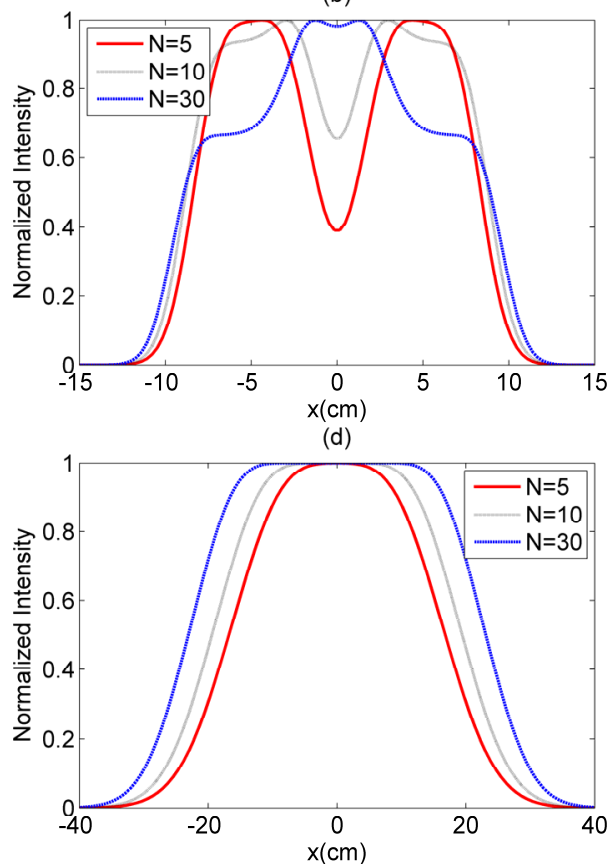

Figure 5. Cross sections $(y=0)$ of the normalized intensity of a RPLMGSMV beam array with $Q=4$ in free space for the different $N$. (a) $z=50 \mathrm{~m},(\mathbf{b}) z=200 \mathrm{~m},(\mathbf{c}) z=400 \mathrm{~m}$, (d) $z=1000 \mathrm{~m}$. 
(a)

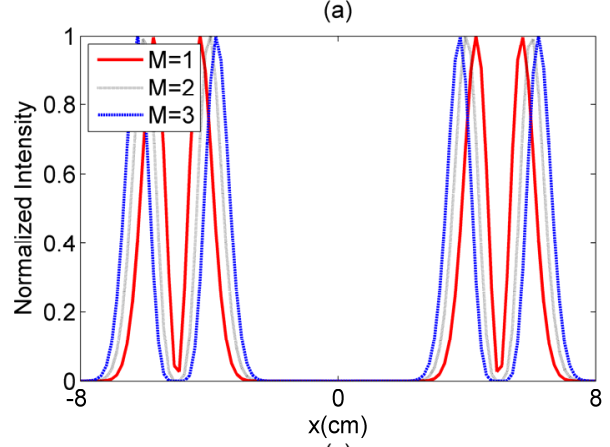

(c)

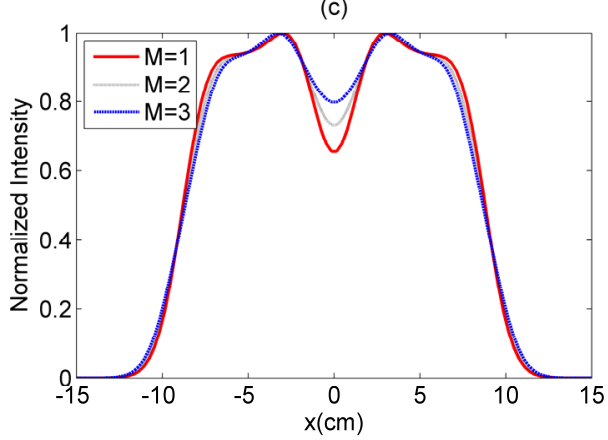

(b)

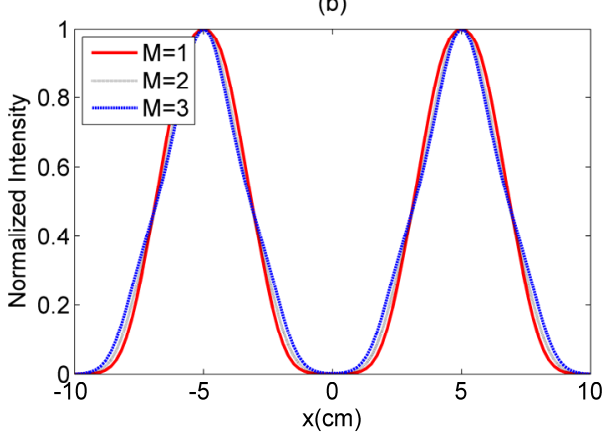

(d)

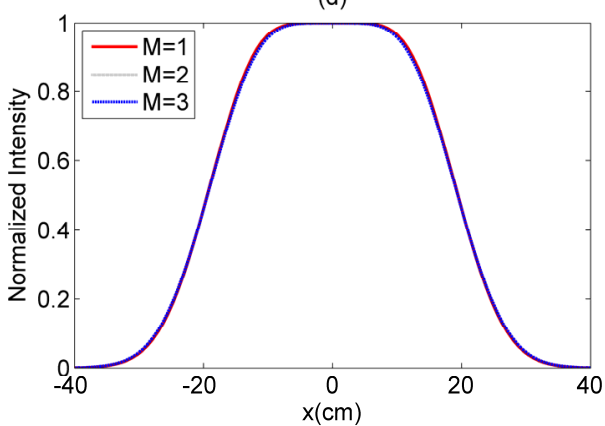

Figure 6. Cross sections $(y=0)$ of the normalized intensity of a RPLMGSMV beam array with $Q=4$ in free space for the different M. (a) $z=0 \mathrm{~m},(\mathbf{b}) z=100 \mathrm{~m},(\mathbf{c}) z=200 \mathrm{~m},(\mathbf{d}) z=1000 \mathrm{~m}$.

Figure 7 illustrates the average intensity of a RPLMGSMV beam array with $Q=4$ propagating in free space and atmospheric turbulence for different $C_{n}^{2}$. As can be seen that in Figure $7 \mathrm{~b}$, the flatness of the flat-topped profile obtained at the longer distance is poorer than a RPLMGSMV beam array in free space, and the larger the $C_{n}^{2}$ is, the poorer the flatness of flat-topped profile is. The phenomenon where the flat-topped profile is becoming poor in atmospheric turbulence can be explained by the influences of atmospheric turbulence.

(a)

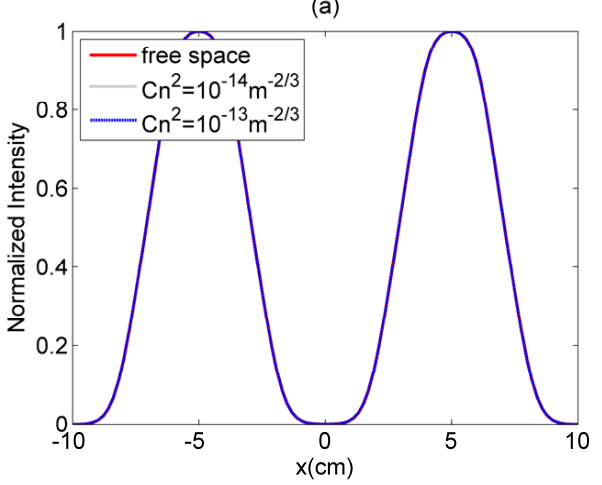

(b)

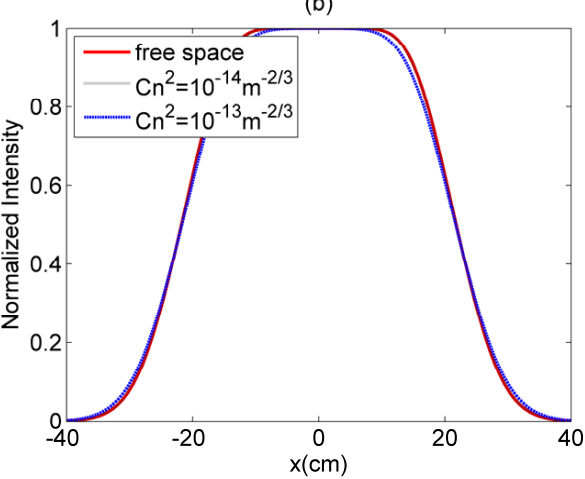

Figure 7. Cross sections of a RPLMGSMV beam array with $Q=4$ in atmospheric turbulence for the different $C_{n}^{2}$. (a) $z=100 \mathrm{~m},(\mathbf{b}) z=1000 \mathrm{~m}$.

Figure 8 gives the cross-sections of degree of coherence of a RPLMGSMV beam array with $Q=5$ in free space (Figure $8 \mathrm{a}-\mathrm{c}$ ) and atmospheric turbulence at $z=1000 \mathrm{~m}$ for the different $M, \sigma, N$ and $C_{n}^{2}$. From Figure $8 \mathrm{a}, \mathrm{b}$, it is seen that the coherence properties of a RPLMGSMV beam array can be affected by the $M$ and $\sigma$. Meanwhile, the effects of total number $N$ on the coherence distribution is not found (Figure 8c). Further, when $x_{1}-x_{2}$ is smaller, the influences of $M$ and $\sigma$ are less. In the analysis of the influences of atmospheric turbulence on coherence, it is seen that the coherence distribution of a RPLMGSMV beam array in atmospheric turbulence with larger $C_{n}^{2}$ will have the Gaussian distribution (Figure 8d). Meanwhile, the same beam in free pace will have the irregular 
coherence distribution. To view the influences of $z$ on coherence distribution, the coherence of a RPLMGSMV beam array with $Q=5$ at the different distance $z$ is illustrated in Figure 9 . One can see from Figure 9 that a RPLMGSMV beam array at the longer propagation distance will have more regular coherence distribution. One can conclude that the spectral degree of coherence of a RPLMGSMV beam array in atmospheric turbulence will have a Gaussian distribution at longer distance.

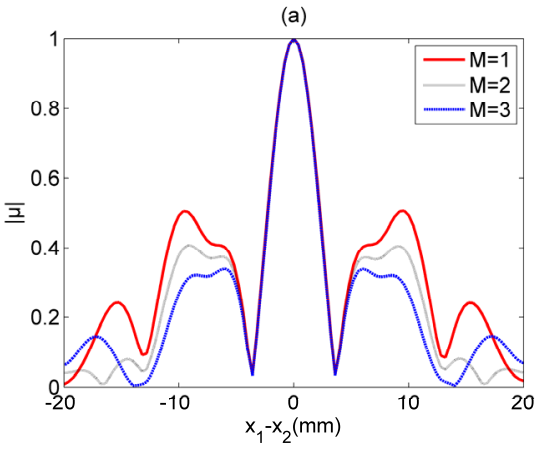

(c)

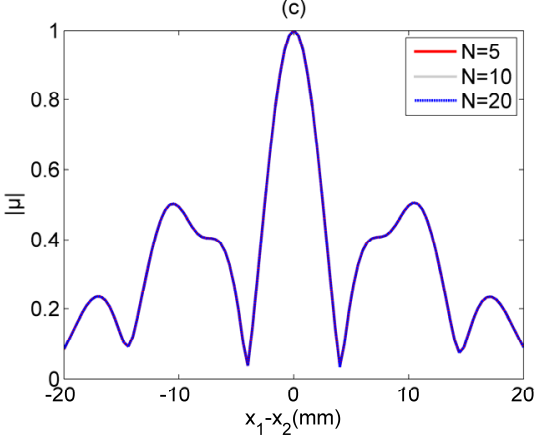

(b)

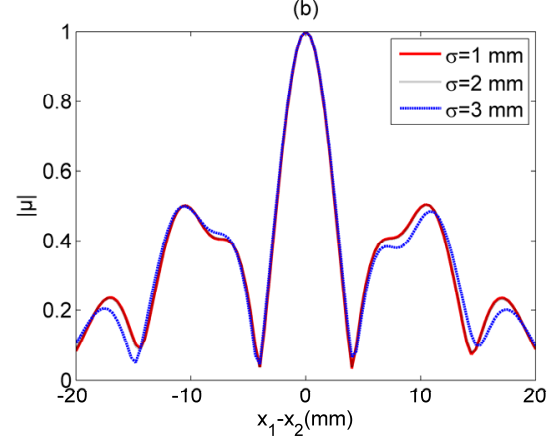

(d)

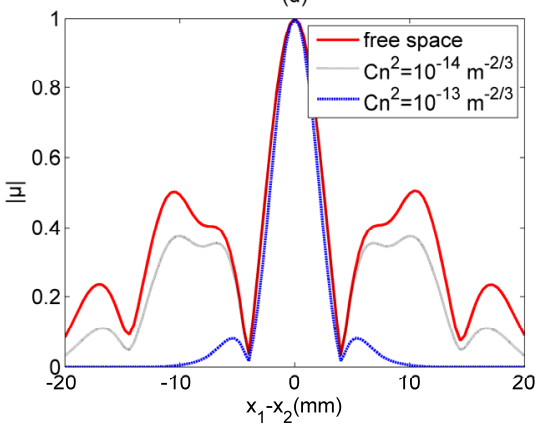

Figure 8. Modulus of the coherence of a RPLMGSMV beam array with $Q=5$ in the free space and atmospheric turbulence at the distance $z=1000 \mathrm{~m}$. (a) different $M,(\mathbf{b})$ different $\sigma,(\mathbf{c})$ different $N$, (d) different $C_{n}^{2}$.

(a)

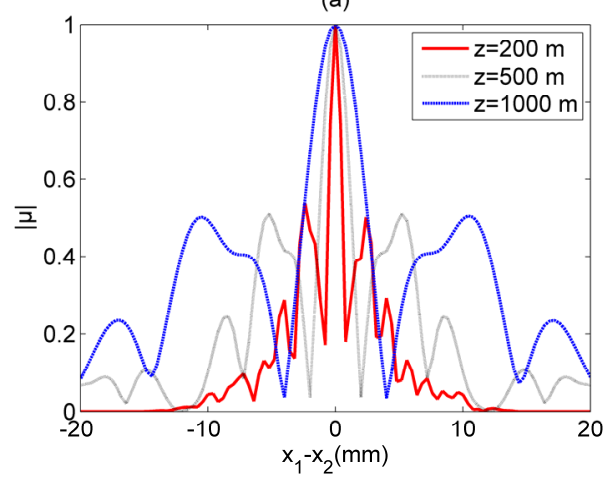

(b)

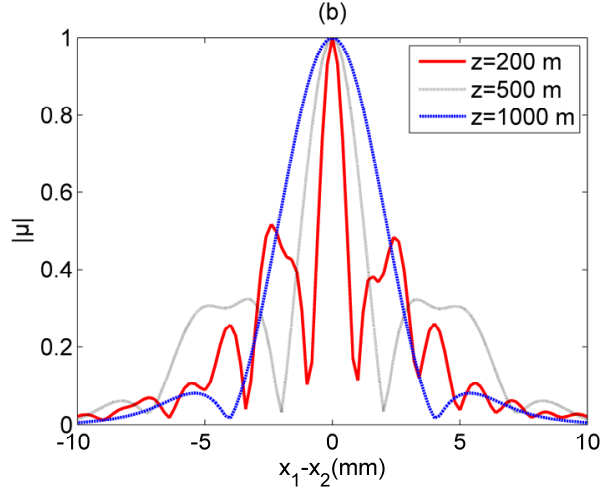

Figure 9. Modulus of the coherence of a RPLMGSMV beam array with $Q=5$ for the different distance $z$. (a) free space, (b) atmospheric turbulence.

\section{Conclusions}

In this paper, the analytical description of a RPLMGSMV beam array generated by MGSM sources is introduced and analyzed. Based on the extended Huygens-Fresnel integral, the CSD of a RPLMGSMV beam array propagating in atmospheric turbulence is derived. The evolutions of intensity and coherence properties of a RPLMGSMV beam array propagating in free space and atmospheric turbulence are analyzed in detail. It is seen that a RPLMGSMV beam array propagating in free space can gradually lose the initial intensity distribution of beamlets, and evolve from the beam array into a beam with 
a flat-topped profile due to the action of MGSM source as $z$ increases. In the far field, when the total number $\mathrm{N}$ is larger or the coherence length $\sigma$ is smaller, the flatness of flat-topped profile of a RPLMGSMV beam array will be better, this is useful for the free space optical communication. When a RPLMGSMV beam array propagates in atmospheric turbulence, the flatness of the flat-topped profile can be dominated by the atmospheric turbulence, the flatness will become poor. It is also found that the coherence distribution of a RPLMGSMV beam array in atmospheric turbulence will have Gaussian distribution at the longer distance.

Author Contributions: Data curation, J.Z. and X.M.; writing—original draft preparation, J.Z., G.W., Y.W., and D.L.; writing-review and editing, H.Z. and H.Y.; supervision, G.W., Y.W., and D.L.; project administration, G.W. and D.L. All authors have read and agreed to the published version of the manuscript.

Funding: This research was supported by National Natural Science Foundation of China [11604038, 11875096, 11404048], and the Fundamental Research Funds for the Central Universities [3132020175].

Acknowledgments: The authors express their appreciation to the anonymous reviewers for their valuable suggestions.

Conflicts of Interest: The authors declare no conflict of interest.

\section{References}

1. Wang, F.; Liu, X.L.; Cai, Y.J. Propagation of partially coherent beam in turbulent atmosphere: A review. Prog. Electromagn. Res. 2015, 150, 123-143. [CrossRef]

2. Peleg, A.; Moloney, J.V. Scintillation index for two Gaussian laser beams with different wavelengths in weak atmospheric turbulence. J. Opt. Soc. Am. A Opt. Image Sci. Vis. 2006, 23, 3114-3122. [CrossRef] [PubMed]

3. Huang, Y.P.; Zhao, G.P.; Gao, Z.H.; He, D. A comparative study of standard and elegant Hermite-Gaussian beams propagating through turbulent atmosphere. J. Mod. Opt. 2011, 58, 1239-1245. [CrossRef]

4. Boufalah, F.; Dalil-Essakali, L.; Nebdi, H.; Belafhal, A. Effect of turbulent atmosphere on the on-axis average intensity of Pearcey-Gaussian beam. Chin. Phys. B 2016, 25, 064208. [CrossRef]

5. Zhou, G.Q. Propagation of a radial phased-locked Lorentz beam array in turbulent atmosphere. Opt. Express 2011, 19, $24699-24711$. [CrossRef]

6. Tang, M.M.; Zhao, D.M. Regions of spreading of Gaussian array beams propagating through oceanic turbulence. Appl. Opt. 2015, 54, 3407-3411. [CrossRef]

7. Lu, L.; Wang, Z.Q.; Zhang, J.H.; Zhang, P.F.; Qiao, C.H.; Fan, C.Y.; Ji, X.L. Average intensity of M x N Gaussian array beams in oceanic turbulence. Appl. Opt. 2015, 54, 7500-7507. [CrossRef]

8. Liu, Z.; Wei, H.Y.; Cai, D.M.; Jia, P.; Zhang, R.; Li, Z.J. Spiral spectrum of Laguerre-Gaussian beams in slant non-Kolmogorov atmospheric turbulence. Optik 2017, 142, 103-108. [CrossRef]

9. Yin, X.; Zhang, L.C. Quantum polarization fluctuations of an Airy beam in turbulent atmosphere in a slant path. J. Opt. Soc. Am. A 2016, 33, 1348-1352. [CrossRef]

10. Dong, K.Y.; Dong, Y.; Song, Y.S.; Chang, S. The properties of anomalous hollow beam propagating in the slant atmosphere. Optik 2018, 172, 1040-1046. [CrossRef]

11. Eyyuboğlu, H.T.; Cai, Y. Non-Kolmogorov spectrum scintillation aspects of dark hollow and flat topped beams. Opt. Commun. 2012, 285, 969-974. [CrossRef]

12. Liu, Y.; Zhang, K.; Chen, Z.; Pu, J. Scintillation index of double vortex beams in turbulent atmosphere. Optik 2019, 181, 571-574. [CrossRef]

13. Zhang, Y.; Zhou, X.; Yuan, X. Performance analysis of sinh-Gaussian vortex beams propagation in turbulent atmosphere. Opt. Commun. 2019, 440, 100-105. [CrossRef]

14. Huang, Y.; Yuan, Y.S.; Liu, X.L.; Zeng, J.; Wang, F.; Yu, J.Y.; Liu, L.; Cai, Y.J. Propagation of optical coherence vortex lattices in turbulent atmosphere. Appl. Sci. 2018, 8, 2476. [CrossRef]

15. Zhang, L.C.; Yin, X.; Zhu, Y. Polarization fluctuations of partially coherent Hermite-Gaussian beams in a slant turbulent channel. Optik 2014, 125, 3272-3276. [CrossRef]

16. Wang, D.; Wang, F.; Cai, Y.; Chen, J. Evolution properties of the complex degree of coherence of a partially coherent LaguerreGaussian beam in turbulent atmosphere. J. Mod. Opt. 2012, 59, 372-380. [CrossRef]

17. Gao, M.; Gong, L.; Wu, P.L. Depolarization characteristics of incompletely polarized and partially coherent laser beams in slant atmospheric turbulence. Optik 2014, 125, 4860-4863. [CrossRef]

18. Xu, Y.G.; Dan, Y.Q.; Zhang, B. Spreading and M-2-factor based on second-order moments for partially-coherent anomalous hollow beam in turbulent atmosphere. Optik 2016, 127, 4590-4595. [CrossRef] 
19. Wang, F.; Li, J.; Martinez-Piedra, G.; Korotkova, O. Propagation dynamics of partially coherent crescent-like optical beams in free space and turbulent atmosphere. Opt. Express 2017, 25, 26055-26066. [CrossRef]

20. Yousefi, M.; Kashani, F.D.; Mashal, A. Analyzing the average intensity distribution and beam width evolution of phase-locked partially coherent radial flat-topped array laser beams in oceanic turbulence. Laser Phys. 2017, 27, 026202. [CrossRef]

21. Kashani, F.D.; Yousefi, M. Analyzing the propagation behavior of coherence and polarization degrees of a phase-locked partially coherent radial flat-topped array laser beam in underwater turbulence. Appl. Opt. 2016, 55, 6311-6320. [CrossRef] [PubMed]

22. Mao, Y.H.; Mei, Z.R.; Gu, J.G. Propagation of Gaussian Schell-model Array beams in free space and atmospheric turbulence. Opt. Laser Technol. 2016, 86, 14-20. [CrossRef]

23. Liu, D.J.; Wang, Y.C.; Yin, H.M. Propagation properties of partially coherent four-petal Gaussian vortex beams in turbulent atmosphere. Opt. Laser Technol. 2016, 78, 95-100. [CrossRef]

24. Mei, Z.; Tong, Z.; Korotkova, O. Electromagnetic non-uniformly correlated beams in turbulent atmosphere. Opt. Express 2012, 20, 26458-26463. [CrossRef]

25. Korotkova, O.; Sahin, S.; Shchepakina, E. Multi-Gaussian Schell-model beams. J. Opt. Soc. Am. A 2012, 29, 2159-2164. [CrossRef]

26. Liu, D.; Wang, Y. Properties of a random electromagnetic multi-Gaussian Schell-model vortex beam in oceanic turbulence. Appl. Phys. B 2018, 124, 176. [CrossRef]

27. Liu, D.; Wang, Y.; Zhong, H.; Wang, G.; Yin, H.; Dong, A. Properties of multi-Gaussian correlated partially coherent anomalous hollow beam propagating in underwater oceanic turbulence. Opt. Laser Technol. 2019, 119, 105604. [CrossRef]

28. Liu, D.-J.; Wang, Y.-C.; Wang, G.-Q.; Yin, H.-M.; Zhong, H.-Y. Properties of multi-Gaussian Schell-model beams carrying an edge dislocation propagating in oceanic turbulence. Chin. Phys. B 2019, 28, 104207. [CrossRef]

29. Xu, H.F.; Zhang, Z.; Qu, J.; Huang, W. Propagation factors of cosine-Gaussian-correlated Schell-model beams in non-Kolmogorov turbulence. Opt. Express 2014, 22, 22479-22489. [CrossRef]

30. Zhu, J.; Li, X.; Tang, H.; Zhu, K. Propagation of multi-cosine-Laguerre-Gaussian correlated Schell-model beams in free space and atmospheric turbulence. Opt. Express 2017, 25, 20071-20086. [CrossRef]

31. Liu, D.; Zhong, H.; Wang, G.; Yin, H.; Wang, Y. Radial phased-locked multi-Gaussian Schell-model beam array and its properties in oceanic turbulence. Opt. Laser Technol. 2020, 124, 106003. [CrossRef]

32. Ma, X.; Liu, D.; Wang, Y.; Yin, H.; Zhong, H.; Wang, G. Propagation of Rectangular Multi-Gaussian Schell-Model Array Beams through Free Space and Non-Kolmogorov Turbulence. Appl. Sci. 2020, 10, 450. [CrossRef]

33. Zhang, Y.T.; Liu, L.; Zhao, C.L.; Cai, Y.J. Multi-Gaussian Schell-model vortex beam. Phys. Lett. A 2014, 378, 750-754. [CrossRef]

34. Wolf, E. Unified theory of coherence and polarization of random electromagnetic beams. Phys. Lett. A 2003, 312, $263-267$. [CrossRef]

35. Jeffrey, A.; Dai, H.H. Handbook of Mathematical Formulas and Integrals, 4th ed.; Academic Press Inc.: Cambridge, MA, USA, 2008. 